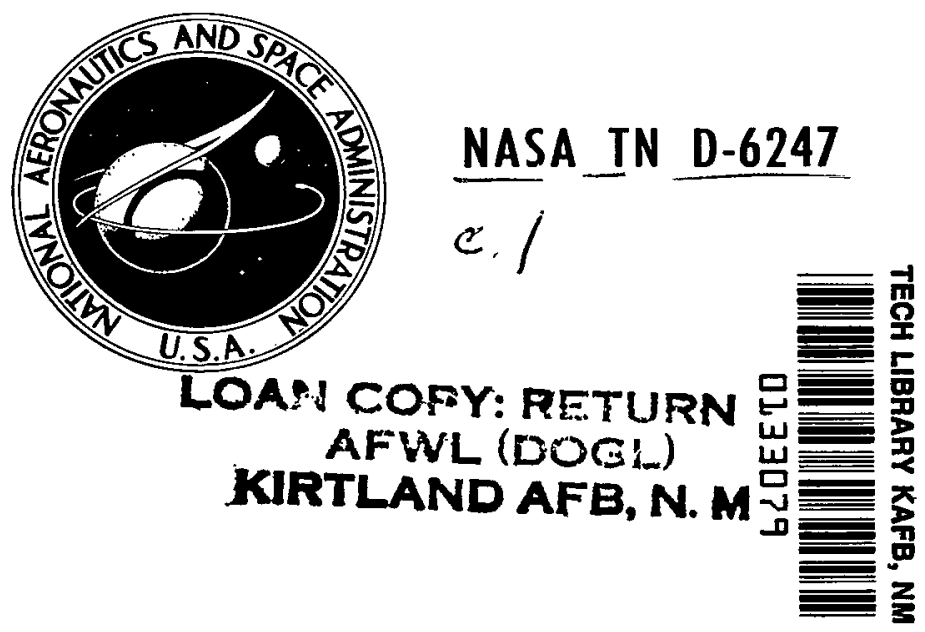

\title{
A SIMULATION STUDY EXPLORING THE EFFECTS OF SENSOR SPATIAL RESOLUTION ON ESTIMATES OF CLOUD COVER FROM SATELLITES
}

by William E. Shenk and Vincent V. Salomonson

Goddard Space Flight Center

Greenbelt, Md. 20771

NATIONAL aERONAUtICS AND SPACE AdMINISTRATION - WASHINGtON, D. C. - MAY 1971 

1. Report No. NASA TTN D-6247
4. Title and Subtitle
A Simulation Study Exploring the Effects of Sensor Spatial Resolution on Estimates of Cloud Cover From Satellites
7. Author(s)
William E. Shenk and Vincent V. Salomonson
9. Performing Organization Name and Address
Goddard Space Flight Center
Greenbelt, Maryland 20771

\section{Government Accession No.}

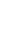

12. Sponsoring Agency Name and Address

National Aeronautics and Space Administration

Washington, D.C. 20546

15. Supplementary Notes 


\section{FOREWORD}

It is the policy of the National Aeronautics and Space Administration to employ, in all formal publications, the international metric units known collectively as the Système Internationale d'Unités and designated SI in all languages. In certain cases, however, utility requires that other systems of units be retained in addition to the SI units.

This document contains data so expressed because the use of the SI equivalents alone would impair communication. The non-SI units, given in parentheses following their computed SI equivalents, are the basis of the measurements and calculations reported here. 
Abstract $\ldots \ldots \ldots \ldots \ldots \ldots \ldots \ldots \ldots \ldots \ldots \ldots \ldots \ldots \ldots \ldots \ldots \ldots \ldots \ldots$

Foreword $\ldots \ldots \ldots \ldots \ldots \ldots \ldots \ldots \ldots \ldots \ldots \ldots \ldots \ldots \ldots \ldots \ldots \ldots \ldots \ldots \ldots \ldots$

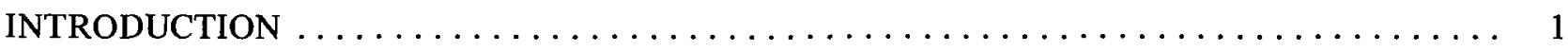

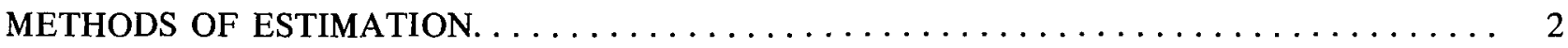

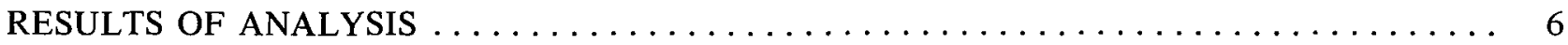

Simulated Cloud Patterns. . . . . . . . . . . . . . . . . . . . . 6

Satellite Data Test . . . . . . . . . . . . . . . . . . . . . . . . 12

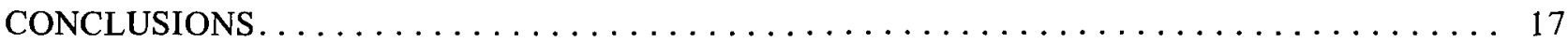

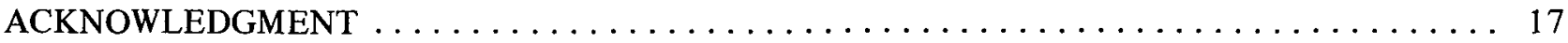

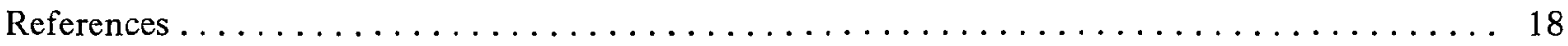




\title{
A SIMULATION STUDY EXPLORING THE EFFECTS OF SENSOR SPATIAL RESOLUTION ON ESTIMATES OF CLOUD COVER FROM SATELLITES
}

\author{
by \\ William E. Shenk \\ and \\ Vincent V. Salomonson \\ Goddard Space Flight Center
}

\section{INTRODUCTION}

Accurate observations of cloud cover in space and time are important in quantitative determinations of the magnitude of the role that clouds contribute to the transfer of energy in the atmosphere. In addition, cloud-cover statistics are also needed in the planning of activities ranging from agricultural enterprises to military and aerospace missions (Sherr et al., 1968; Brown, 1970). Meteorological satellites provide a platform from which this cloud cover over all or large parts of the earth can be observed at least once a day. There are three fundamental factors that must be considered for accurate observations of cloud cover from a satellite using a particular sensor, such as a radiometer or camera, or from the ground by eyesight. These factors are the resolution of the sensing device, the viewing perspective, and the sensor sensitivity or response to clouds within the field of view. For example, the eye of a surface observer has sufficient resolution and sensitivity to detect very small or tenuous clouds, but the perspective of the observer is such that only a small part of the celestial dome is viewed at small local zenith angles (less than $60^{\circ}$ ). As a result of this perspective problem and the natural human tendency for observers to overestimate the percentage of cloud cover (Young, 1967), the amount of cloud cover is nearly always overestimated by a surface observer.

When observations of cloudiness are made from a satellite, the perspective problem is not as severe because the altitude of the satellite above the earth is sufficient to permit the viewing of extensive areas at small nadir angles. On the other hand, the sensitivity and spatial resolution of the sensor are not nearly as good as those of the human eye. For accurate cloud-cover determinations, satellite instrument sensitivity and spatial resolution must be sufficient to detect the presence of individual cloud elements within the field of view. Currently, the sensitivity of satellite instruments responding to visible reflected solar energy is generally adequate for detecting the presence of clouds against a dark background, such as the ocean. Notable exceptions occur when thin cirrus or very scattered and small convective cloud elements are within the field of view. The linear spatial resolution at the subsatellite 
point is about $4 \mathrm{~km}$ for the majority of visual sensors on meteorological satellites. Thus, it is difficult to resolve individual cloud elements in convective cloud fields or small holes in layered cloud fields where the elements and holes are small. The general result is an overestimation of the percentage of the cloud cover because of an integration or blurring of the cloud areas with clear areas.

The intent of this paper is to examine the spatial resolution effect on estimates of cloud cover. Simulated cloud patterns are used for this purpose in order to isolate the resolution effect from the other two fundamental factors and to provide a knowledge of the true cloud-cover percentage.

\section{METHODS OF ESTIMATION}

Three different types of cloud patterns were constructed:

(1) Cloud pattern 1 is a regularly spaced pattern of dots (clouds), all the same size, arranged in rows and columns most nearly representing cloud streets (see Figure 1).

(2) Cloud pattern 2 is a randomly spaced pattern of equal-size dots, most closely simulating a field of randomly spaced cumulus clouds (Figure 2).

(3) Cloud pattern 3 is composed of different cloud sizes and shapes irregularly spaced, representing a real cloud pattern composed of different cloud types and sizes (Figure 3).

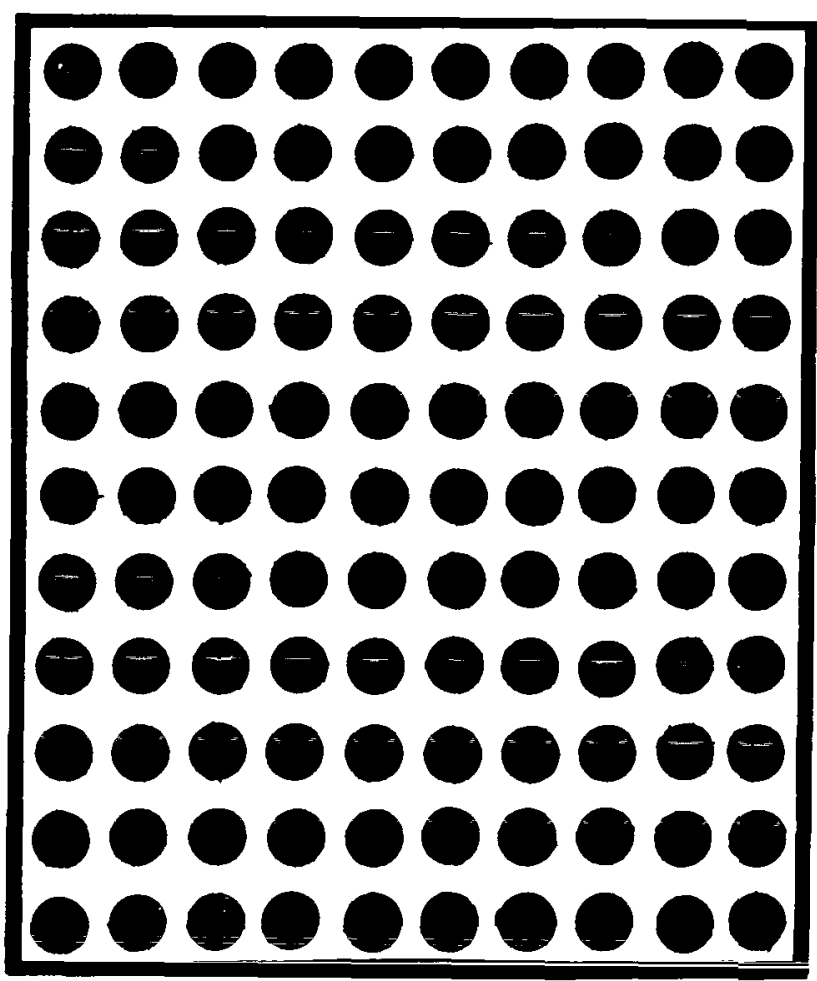

Figure 1-A simulated cloud pattern comprised of regularly spaced dots all the same size arranged in rows and columns (cloud pattern 1).

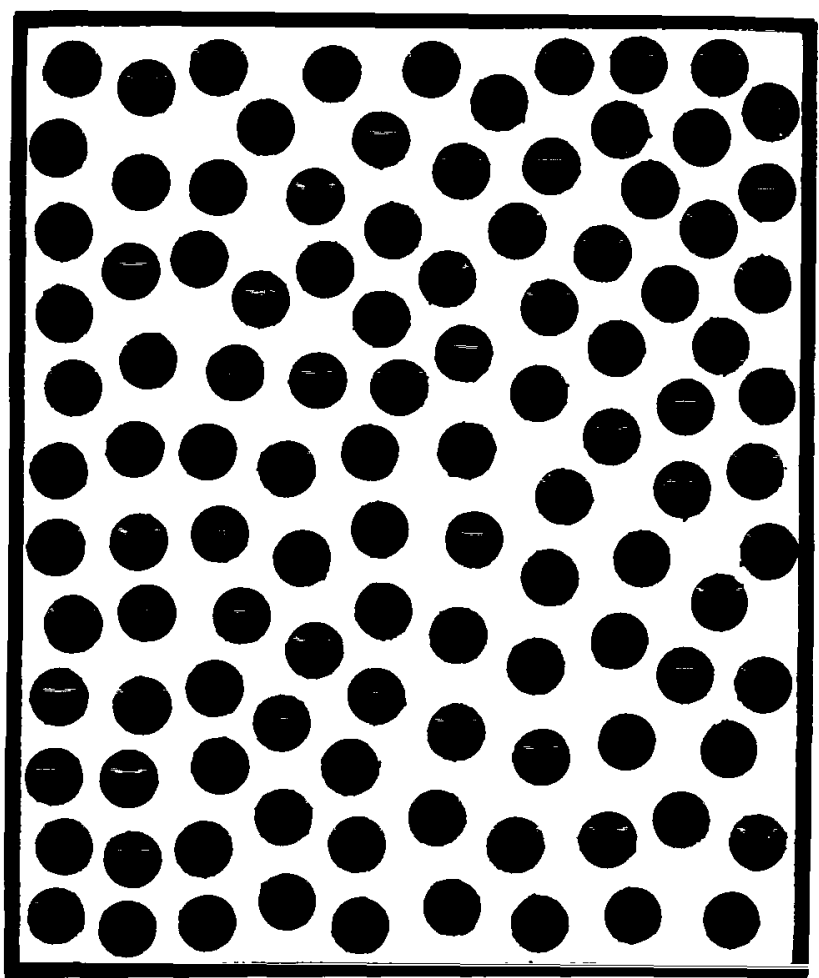

Figure 2-A simulated cloud pattern comprised of randomly spaced dots of equal size (cloud pattern 2). 
The true percentage of cloud cover in each cloud pattern was determined from knowledge of the amount of surface area used in construction of the cloud patterns from dark paper and, in the case of cloud pattern 3 , was checked by planimetry of the cloud areas in the cloud patterns once they were constructed. The cloud cover is approximately 35 percent for each of the cloud patterns shown in Figures 1 through 3. Other cloud fields, containing four different cloud covers from 14 to 72 percent, were generated for all three cloud patterns and, in addition, a 90 percent cloud cover situation was prepared for cloud pattern 3. Also, for cloud patterns 1 and 2, a 5 percent cloud cover was used.

In order to simulate clouds of different size, the diameter of the dots in Figure 2 was multiplied by factors of 0.5 and 2 , respectively, while the cloud cover percentage was held fixed. Figure 4 illustrates the size differences in the dots for cloud pattern 2. Because the cloud sizes were the same within each case for cloud patterns 1 and 2, the average cloud size was known. In the case of cloud pattern 3, the average cloud size $\bar{A}$ was determined from a simple area-weighting computation:

$$
\bar{A}=\frac{1}{n} \sum_{i=1}^{n} A_{i},
$$

where $n$ is the total number of cloud elements and $A_{i}$ is the area of each cloud element $i$.

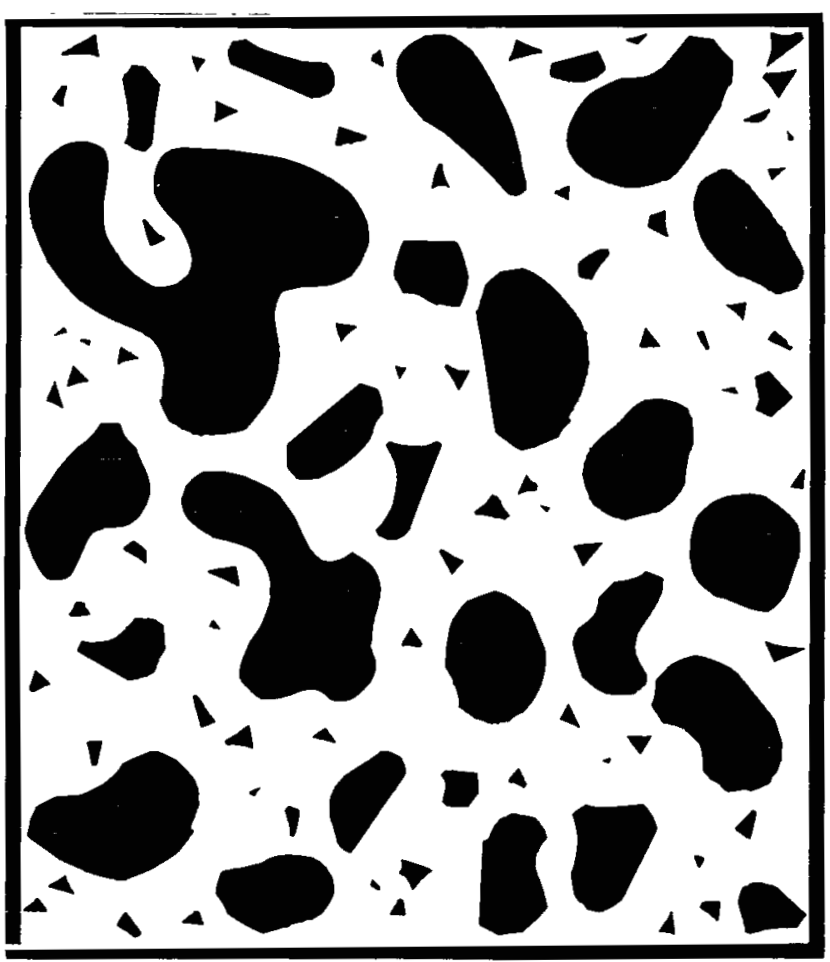

Figure 3-A simulated cloud pattern comprised of randomly spaced elements of different sizes and shapes (cloud pattern 3).
Resolution elements were created on a twodimensional grid, such as the example shown in Figure 5, where each rectangle represents a resolution element. The resolution elements varied in size, depending on the number of elements in each row and column. A $10 \times 15$ element grid contained the smallest number of resolution elements (150) and a $120 \times 180$ grid the largest number $(21,600)$, with 5 grids in between $(20 \times 30$, $40 \times 60,60 \times 90,80 \times 120$, and $100 \times 150$ ). Figure 5 depicts the $10 \times 15$ resolution element grid. Thus, the linear spatial resolution varied by a factor of 12 and the areal spatial resolution by 144. With a linear factor variation of 4 in the cloud-dot diameter, the total range of the ratio of linear cloud diameter to resolution element size is 48 , and the range of the ratio $R$ of areal cloud size to areal resolution element size is about 2300 . In the case of cloud pattern 3 , where the only cloud-size variation considered was a difference in the mean cloud size for each of the six cloud distributions, the ranges of the linear ratio and the areal ratio were 21 and 455, respectively. Table 1 lists values of $R$ for all three cloud patterns and for the full range of resolution element sizes. The 


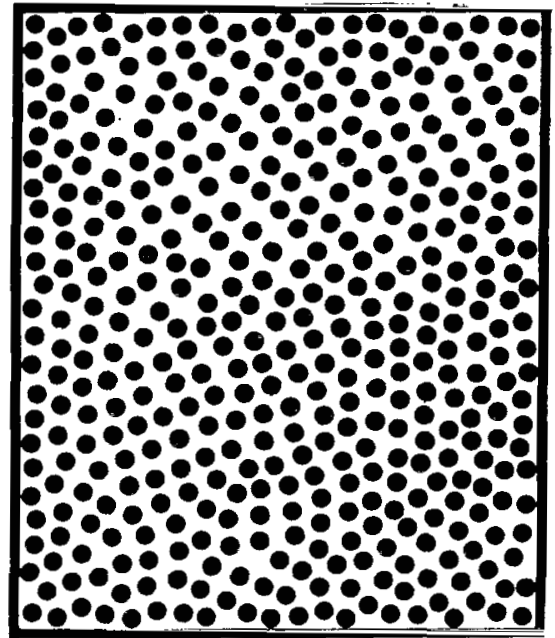

(a)

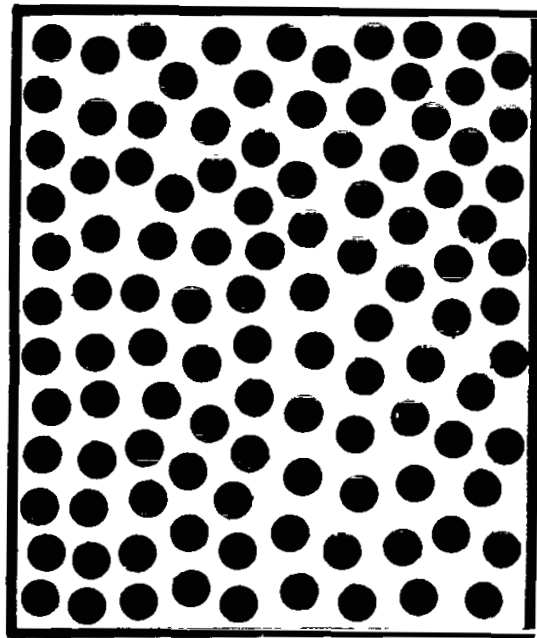

(b)

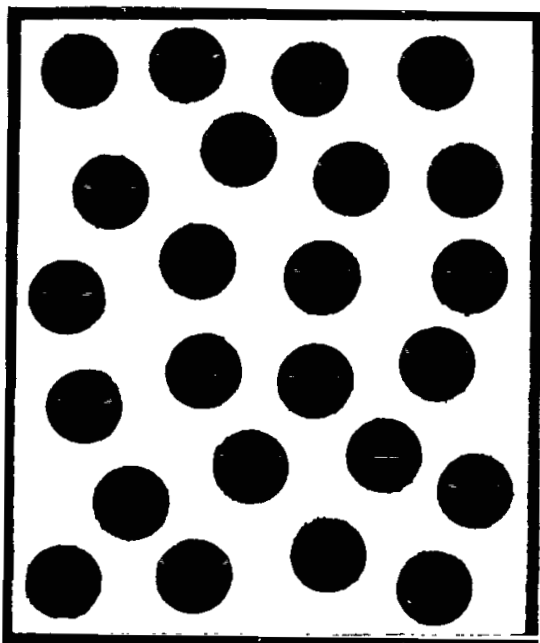

(c)

Figure 4-Three cloud sizes for (a) cloud pattern 2 where the cloud size has been (b) increased by a factor of 2 and (c) increased by a factor of 4 .

table indicates that the total range of $R$ for cloud patterns 1 and 2 varies from a situation where the areal resolution element size is about nine times larger than the average areal size of the clouds to the condition where the areal resolution element is approximately 0.0035 the average areal cloud size. The range of $R$ for cloud pattern 3 is slightly smaller and is contained within the range indicated for cloud patterns 1 and 2 . It was expected that the cloud-cover estimation capability would improve steadily once $R$ became greater than or equal to unity. As
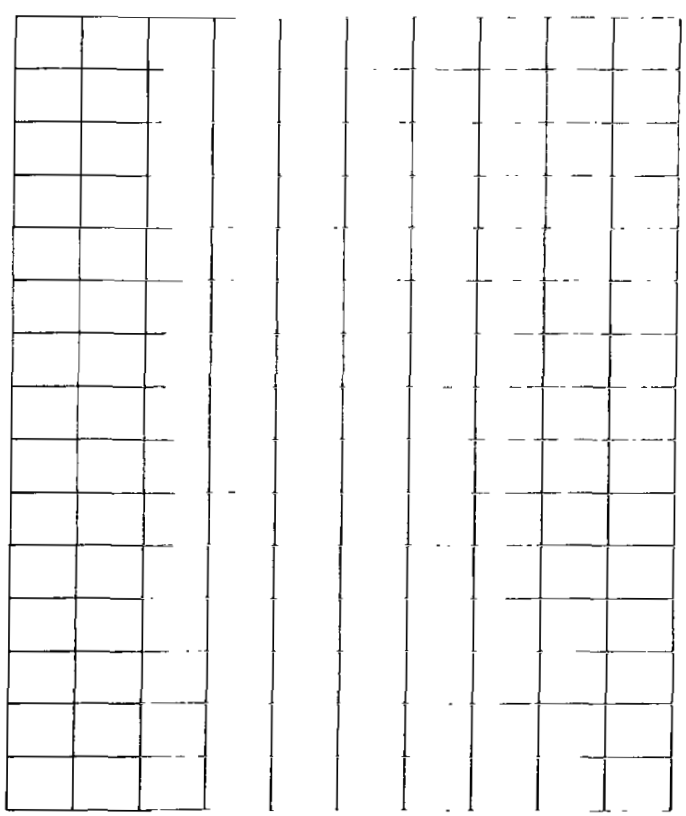

Figure 5-An example of a resolution grid $(10 \times 15$ elements $)$. a result, the range of $R$ for all three cloud patterns covered the anticipated critical zone from 1 to 100 . Table 1 also shows redundancy in $R$ for cloud patterns 1 and 2 between the small, medium, and large dots. For example, $R=7.95$ for the $80 \times 120$ resolution grid with the small dots, the $40 \times 60$ resolution grid with the medium dots, and the $20 \times 30$ grid with the large dots. This redundancy provided a check on the consistency of the cloud-cover calculations for a given $R$ for each cloud pattern.

Through use of the resolution grids, the percentages of cloud cover were determined in two ways. One method was to count every resolution element that contained some portion of a cloud element and consider that resolution element as being completely cloud filled. This is analogous to the satellite situation where a brightness threshold is established slightly above the background brightness (such as the ocean), the number of digitized samples which are above the threshold are counted, and this count is then divided by the total number of samples scanned, which 
Table 1-The ratio $R$ of cloud size to resolution element size for the three different cloud patterns.

\begin{tabular}{|c|c|c|c|c|c|c|}
\hline \multicolumn{7}{|c|}{$\begin{array}{l}\text { Cloud patterns } 1 \text { and } 2 \\
\text { (independent of the cloud cover percentage) }\end{array}$} \\
\hline \multirow{2}{*}{\multicolumn{2}{|c|}{$\begin{array}{l}\text { Resolution element grid } \\
\quad \text { (Rows } \times \text { Columns) }\end{array}$}} & \multicolumn{5}{|c|}{ Dot size } \\
\hline & & \multicolumn{2}{|l|}{ Small } & Medium & \multicolumn{2}{|c|}{ Large } \\
\hline \multicolumn{2}{|l|}{$10 \times 15$} & \multicolumn{2}{|l|}{0.12} & 0.50 & \multicolumn{2}{|c|}{1.99} \\
\hline $20 \times 30$ & & \multicolumn{2}{|c|}{0.50} & 1.99 & \multicolumn{2}{|c|}{7.95} \\
\hline $40 \times 60$ & & \multicolumn{2}{|l|}{1.99} & 7.95 & \multicolumn{2}{|c|}{31.79} \\
\hline $60 \times 90$ & & \multicolumn{2}{|l|}{4.47} & 17.88 & \multicolumn{2}{|c|}{71.53} \\
\hline $80 \times 120$ & & \multicolumn{2}{|l|}{7.95} & 31.79 & \multicolumn{2}{|c|}{127.18} \\
\hline $100 \times 150$ & & \multicolumn{2}{|l|}{12.42} & 49.67 & \multicolumn{2}{|c|}{198.69} \\
\hline $120 \times 180$ & & \multicolumn{2}{|l|}{17.88} & 71.53 & \multicolumn{2}{|c|}{286.11} \\
\hline \multicolumn{7}{|c|}{ Cloud pattern 3} \\
\hline \multirow{2}{*}{$\begin{array}{l}\text { Resolution element grid } \\
\quad \text { (Rows } \times \text { Columns) }\end{array}$} & \multicolumn{6}{|c|}{$\begin{array}{l}\text { Cloud cover } \\
(\%)\end{array}$} \\
\hline & 14 & 21 & 36 & 55 & 71 & 90 \\
\hline $10 \times 15$ & 0.39 & 0.40 & 0.58 & 0.40 & 1.17 & 0.37 \\
\hline $20 \times 30$ & 1.55 & 1.59 & 2.32 & 1.60 & 4.67 & 1.48 \\
\hline $40 \times 60$ & 6.23 & 6.37 & 9.31 & 6.42 & 18.74 & 5.96 \\
\hline $60 \times 90$ & 13.97 & 14.30 & 20.89 & 14.42 & 41.93 & 13.35 \\
\hline $80 \times 120$ & 24.82 & 25.40 & 37.13 & 25.63 & 74.51 & 23.73 \\
\hline $100 \times 150$ & 38.74 & 39.65 & 57.96 & 40.00 & 116.30 & 37.04 \\
\hline $120 \times 180$ & 56.04 & 57.36 & 83.84 & 57.86 & 168.24 & 53.59 \\
\hline
\end{tabular}

yields the percentage of cloud cover. The other method is to subdivide the data into three regimes, where (1) the resolution element is clear, (2) the resolution element is partially cloud filled, and (3) the resolution element is completely cloud filled. A weighting factor of 1 is multiplied by the number of partially cloud-filled resolution elements and a weighting factor of 2 by the number of completely cloud-filled resolution elements. The summation of these resolution elements multiplied by their appropriate weighting factors is divided by twice the total number of resolution elements to yield the percentage of cloud cover. This second method assumes a cloud cover of 50 percent for every partially filled resolution element. The second method, as applied to satellite data, would resemble a procedure establishing two thresholds, one each at the low and high ends of the brightness range. Brightness values at or below the lower threshold would indicate cloudless conditions, whereas brightness values at or above the upper threshold would coincide with the resolution element's being completely cloud filled. All brightness values between the thresholds would then be assumed to be associated with partially cloud-filled resolution elements. 


\section{RESULTS OF ANALYSIS}

\section{Simulated Cloud Patterns}

A typical example of a curve of the estimated percentage of cloud cover (obtained by the first method for cloud cover estimation) versus $R$ for a given true percentage of cloud cover is shown in Figure 6. Due to the fact that an integral number of dots was required to construct cloud pattern 1 , the percentages of cloud cover are not quite equal for the small, medium, and large dot categories. The scatter in Figure 6 is very small for the three different cloud patterns, being less than 3 percent for all values of $R$ when consideration is given for the differences in cloud cover between the cloud fields within cloud patterns 1 and 2 . Thus, the percentage of cloud cover estimated was nearly independent of the cloud pattern that was used to obtain the percentage of cloud cover. Also, the scatter shows that for a given $R$ within cloud patterns 1 and 2 the different dot sizes and the human judgment factor in estimations of the percentage of cloud cover from simulated cloud pattern to pattern had little or no effect on the results. Figure 6 also shows that an $R$ value of 200 is required before the estimated cloud cover becomes within 10 percent of the true cloud cover. Even for an $R$ value of about 300 (the upper limit of the data) the difference between the estimated and the true cloud cover is still 8 percent. For a value of $R$ near 10, the estimated cloud cover is 91 percent for a true average cloud-cover value (for all cloud patterns) of 55 percent. Thus, it is clear that the areal size of a resolution element has to be at least 100 times smaller than the average areal cloud size in this case before reasonable agreement appears between the estimated and true percentages of cloud cover. The results

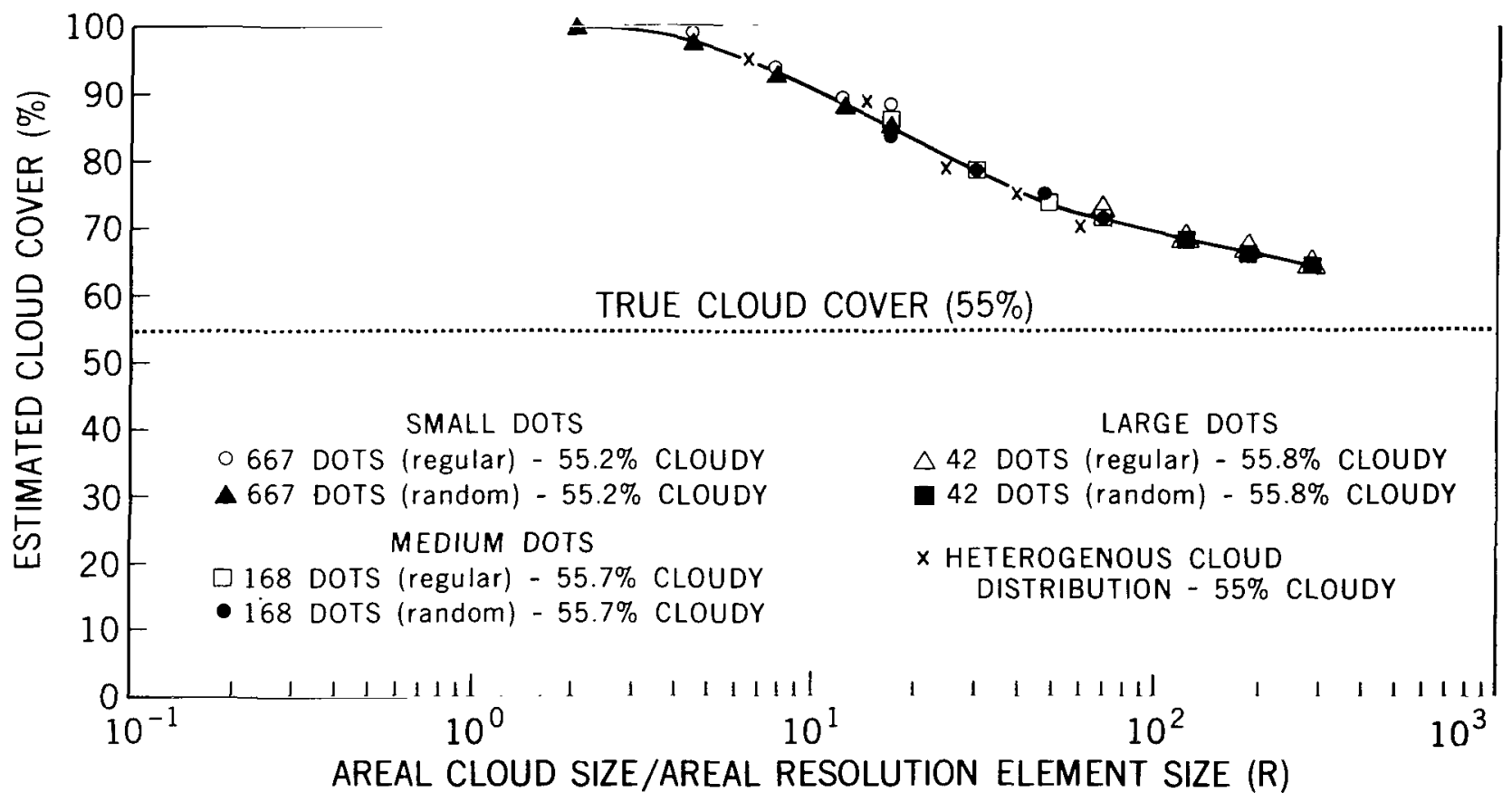

Figure 6-Estimated cloud cover (from method 1) for a true civiu cover of about 55 percent as a function of the areal cloud size/areal resolution element size ratio $(R)$ for the three simulated cloud patterns. 
for the other curves prepared from the other cloud fields (different percentages of cloud cover) for each cloud pattern are very similar to the example presented in Figure 6. Cloud patterns 1 and 2 agree very well with cloud pattern 3 for all percentages of cloud cover.

Figure 7 shows the resultant nomogram that emerged from the curves prepared for each true percentage of cloud cover similar to Figure 6 . The portions of the curve beyond the limits of the data were extrapolated, i.e., the regions from 0 to 5 percent and 90 to 100 percent cloud cover for all values of $R$, and for all cloud-cover percentages for $R<0.125$ and $R>286$. Many of the results discussed in connection with Figure 6 are also present in this more general nomogram. For true cloud covers between about 30 and 70 percent, an $R$ value of 100 is associated with a flattening of the true percentage curves as the estimated percentage of cloud cover becomes reasonably close to the true percentage of cloud cover. The flattening of the true cloud-cover percentage curves occurs near an $R$ value of 10 for less than 30 percent true cloud cover and near an $R$ value of 1000 for greater than 70 percent cloud cover. A somewhat surprising result is the amount of error in estimates of the cloud-cover percentages when $R=1$. For example, for $R=1$ when the true cloud cover is 20 percent, the estimated cloud cover is 70 percent. A value of $R=1$ is reasonable in the tropics, where the average cumuliform cloud size can be about $4 \mathrm{~km}$ and the resolution of the satellite sensor is the same. For $R<1$, the cloudcover percentage estimates continue to deviate further from the true values, and at $R=0.1$, even a 14 percent cloud cover appears to be totally cloud covered.

A noticeable bulge in the true cloud-cover curves of Figure 7 becomes more pronounced for small true percentages of cloud cover. This bulge probably becomes even more pronounced for true cloud

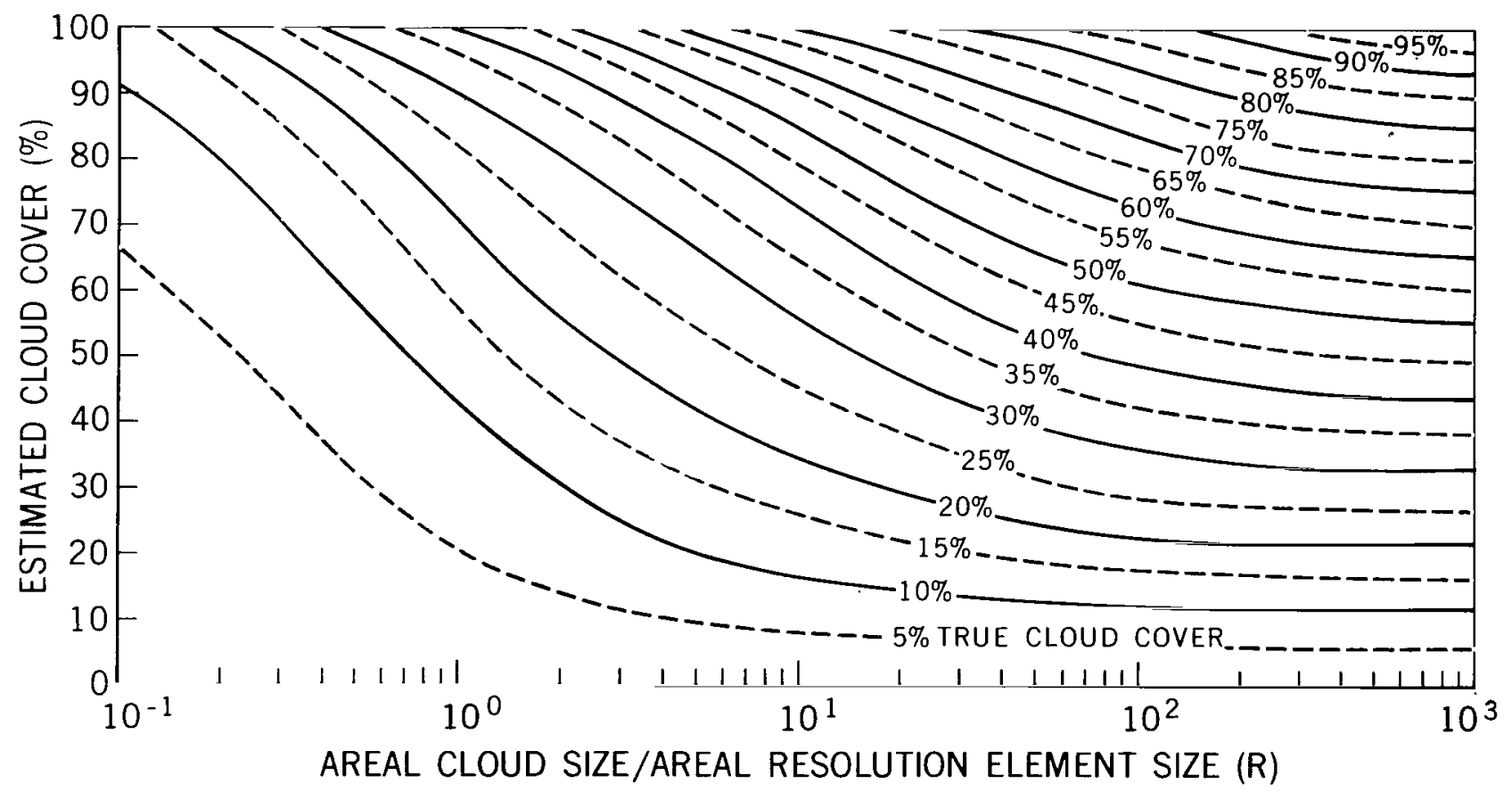

Figure 7-A nomogram generated from the three simulated cloud patterns showing estimates of cloud cover (from method 1) as a function of $R$ and true cloud cover. 
covers less than 5 percent. The estimated cloud-cover percentages still do not match the true cloudcover percentages for values of $R$ near 1000. Perfect estimates cannot occur with this first method of cloud-cover/clear-area estimation, because there will always be partially filled resolution elements that will be counted as completely filled.

The nomogram presented in Figure 7 shows that considerable overestimates of cloudiness, or underestimates of the clear-sky percentage, occur when method 1 estimation criteria are employed. In the latter instance, this result gives some useful insight into how frequently one can expect to find cloud-free observations in various cloud-cover situations. Insight such as this is relevant in assessment of the probability of obtaining observations for remote detection of the vertical temperature structure in the atmosphere from satellite-borne spectrometers (Hanel and Conrath, 1969; Wark and Hilleary, 1969; Stamm and Von der Haar, 1970) and when observations are made of geological, agricultural, or oceanographic features from satellites where it is highly desirable that an observation be free of cloud contamination. From the converse of a previous example, the nomogram in Figure 7 shows that when $R=1$ and the true clear-sky fraction is 80 percent, only 30 percent of the observations will be cloud free. For existing temperature-sounding instruments, $R>10$ would occur infrequently.* Since the earth is 50 to 60 percent cloud free, Figure 7 indicates that one could expect less than 20 percent of the observations made by existing temperature-sounding instruments to be cloud free. This conclusion agrees with results presented in a study by Williamson and Warnecke. $\dagger$ On the first Earth Resources Technology Satellite (ERTS) scheduled for launch in the spring of 1972, the spatial resolution of the return beam vidicon (RBV) camera and the multi-spectral sensor (MSS) will be better than $0.1 \mathrm{~km}$. Therefore, $R$ will be larger than 100 most of the time, and, consequently, Figure 7 predicts that the number of cloud-free observations will comprise a large fraction of the available clear-sky area within the field of view of the ERTS instruments.

The nomogram in Figure 7 can be used to improve cloud-cover/clear-area estimates, provided that $R$ is known. It is not necessary that $R$ be known with great precision, since $R$ varies logarithmically along the abscissa. The estimated cloud-cover percentage can be entered into the nomogram along with some knowledge of $R$ to provide a better estimate of the true percentage of cloud cover. Even for an estimated cloud cover of 100 percent, improved knowledge of the true cloud cover can be ascertained with an estimate of $R$. Variations in the amount of reflected light over the seemingly complete cloud cover could lead to a determination of cloud type and also to an estimate of average cloud size (Conover, 1962, 1963).

Figure 8 is a typical curve of $R$ versus estimated percentage of cloud cover by the second method of estimating the percentage of cloud cover. Since the partially cloud-filled resolution elements are assumed to be 50 percent cloud filled, the estimated percentage of cloud cover is 50 percent for large resolution elements where there are no cloud-free or completely cloud filled resolution elements. As the resolution increases, the percentage of the estimated cloud cover reflects a rapidly increasing number of cloud-free resolution elements, since the true cloud cover is 20 percent. At $R=10$, the percentage

\footnotetext{
*The spatial resolutions of the Satellite Infrared Spectrometer (SIRS) and the Infrared Interferometer Spectrometer (IRIS) on Nimbus IV are $230 \mathrm{~km}$ and $90 \mathrm{~km}$, respectively.

†Williamson, E. J., and Warnecke, G., "The Influence of Cloud Distribution on the Global Coverage of Remote Atmospheric Sounding Systems," unpublished report.
} 


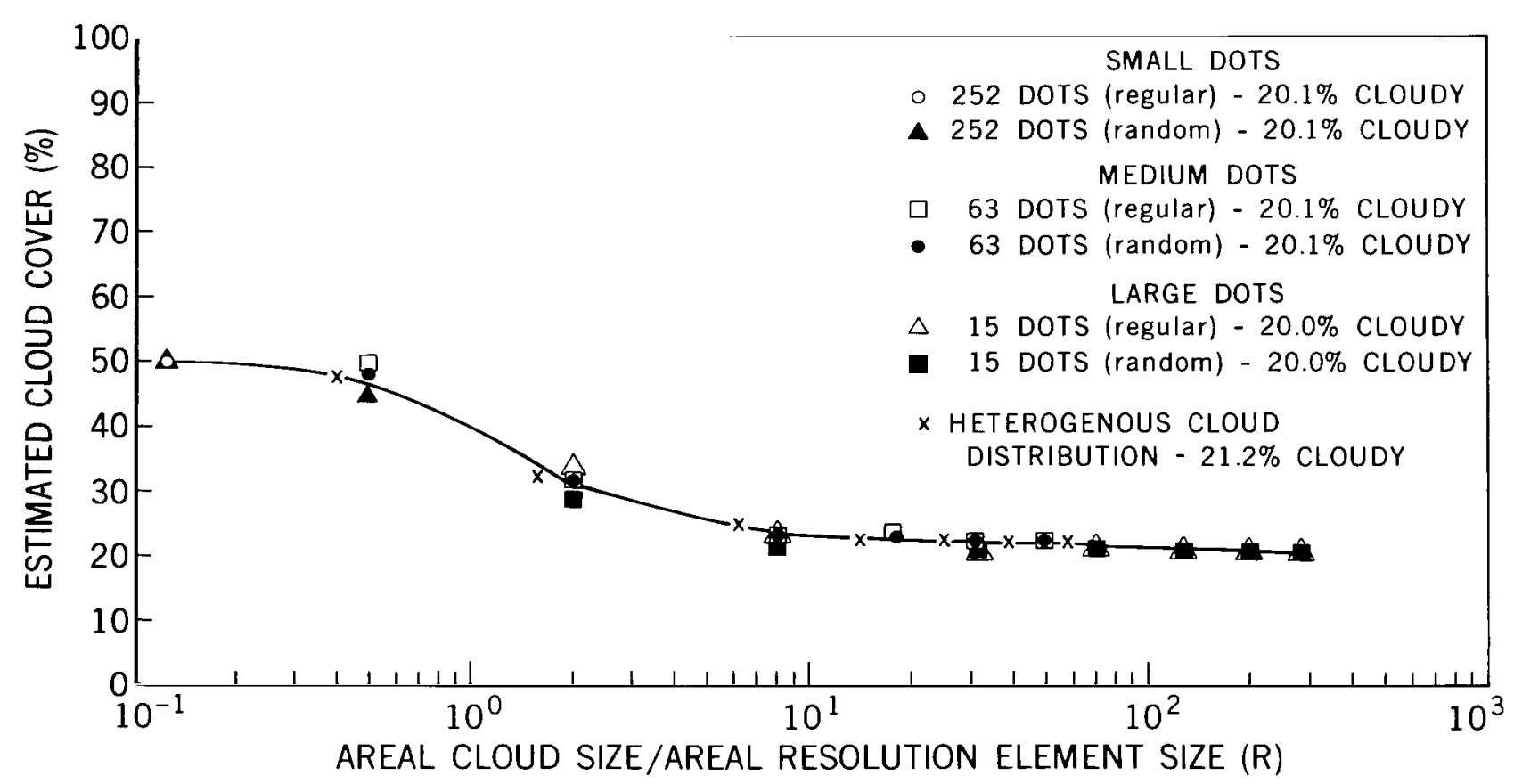

Figure 8-Estimated cloud cover (from method 2) for approximately 20 percent true cloud cover as a function of $R$ for the three simulated cloud patterns.

of cloud cover estimated by the second method was close to the true percentage of cloud cover. For this particular percentage of cloud cover, the results for all three of the cloud patterns are similar over the full range of $R$. A scatter of \pm 2.5 percent about the mean in the estimate of the percentage of cloud cover occurred from $R=0.5$ to $R=2$; beyond these limits the scatter decreased to less than \pm 1 percent at $R=0.1$ and $R \geqslant 8$. However, when the true cloud cover was greater than 35 percent, differences began to appear between the results for cloud patterns 1 and 2 and cloud pattern 3 . Figure 9 illustrates these differences, which are noticeable between $R=0.5$ and $R=25$ for a true cloud cover of about 55 percent. The estimated cloud covers for cloud pattern 3 begin increasing above 50 percent at a smaller $R$ than for cloud patterns 1 and 2 . This effect is probably due to the larger individual simulated clouds that are present in cloud pattern 3 . With large resolution elements, there are few, if any, clear areas to compensate for the completely filled resolution elements over the large cloud elements. This results in estimated cloud covers of over 50 percent. In the case of cloud patterns 1 and 2, there are also few, if any, clear resolution elements, but the uniform cloud size prevents the presence of the large cloud elements that would cause a substantial percentage of the resolution elements to be completely cloud filled. The result is that almost every resolution element is partially filled, which means that the total estimated cloud cover is close to 50 percent.

Separate nomograms similar to Figure 7 were prepared for cloud patterns 1 and 2 and cloud pattern 3 as a result of the differences in estimated percentage of cloud cover between the cloud patterns for a given $R$. The first nomogram, for cloud patterns 1 and 2, is shown in Figure 10. An important feature of this nomogram is that there is an upper boundary of 78 percent cloud cover imposed by the geometry of nonoverlapping dots placed side by side. The nomogram reveals that for $R \geqslant 10$, for 


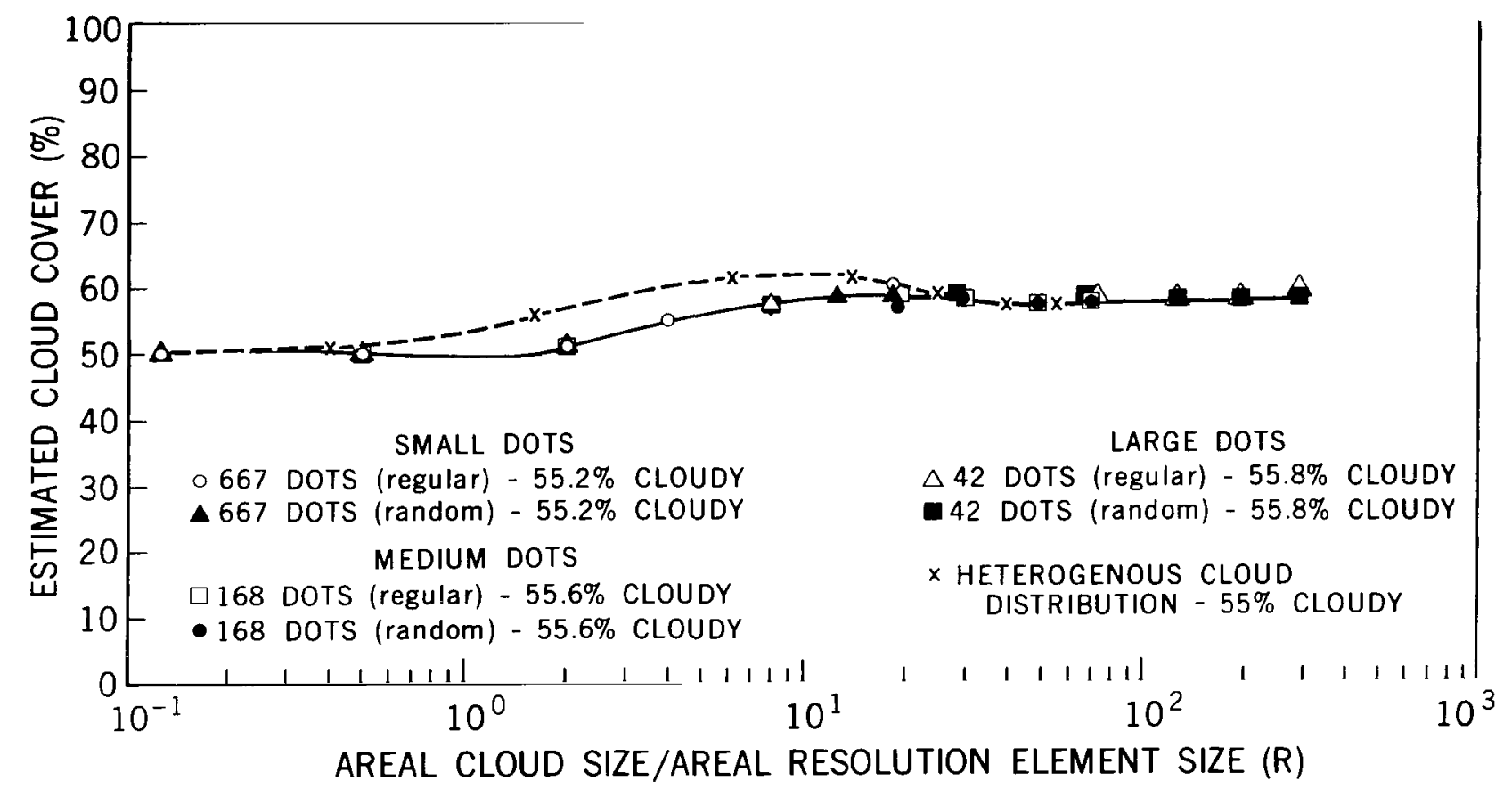

Figure 9-Estimated cloud cover (from method 2) for approximately 55 percent true cloud cover as a function of $R$ for the three simulated cloud patterns.

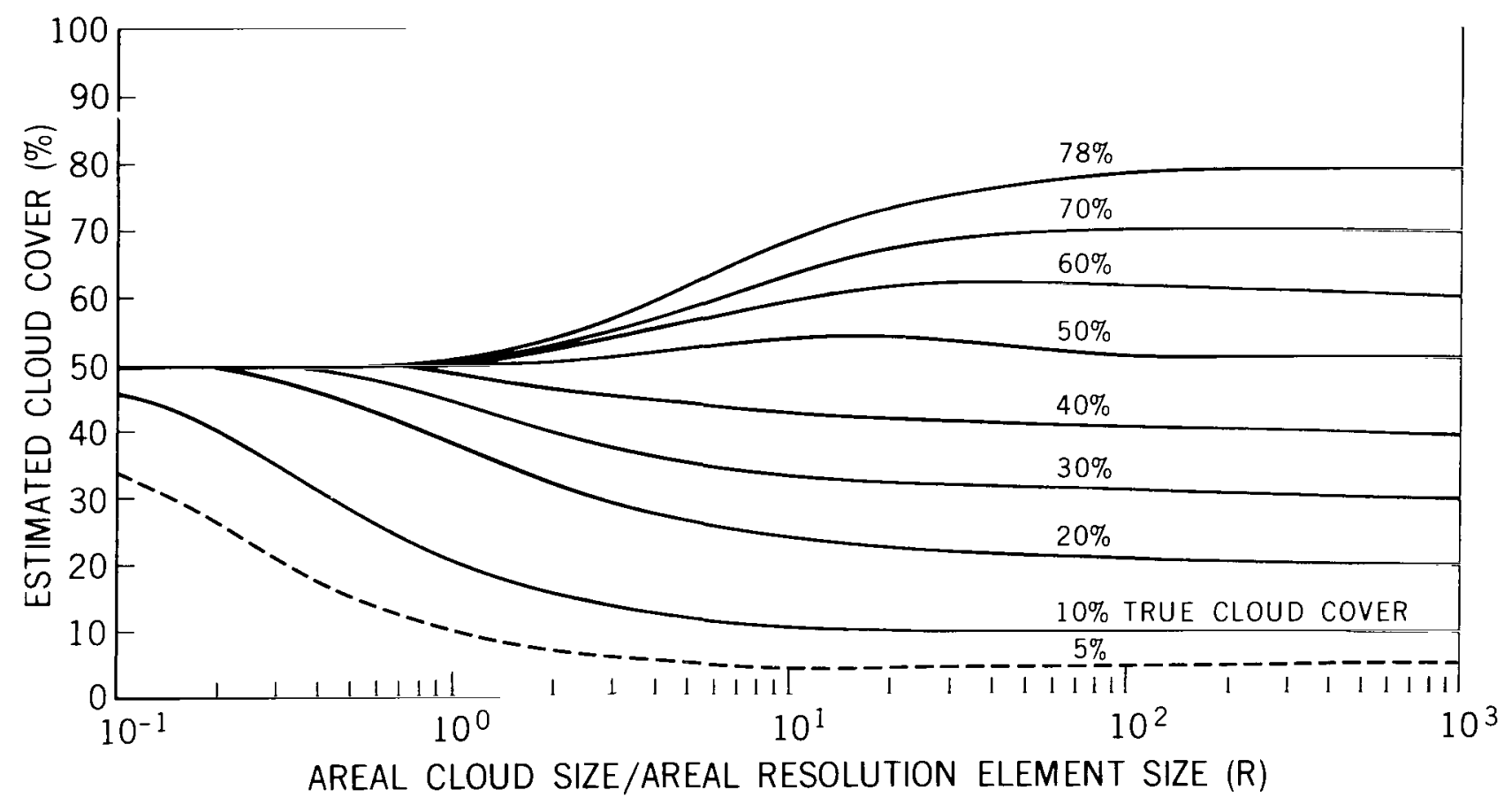

Figure 10-A nomogram generated from simulated cloud patterns 1 and 2, from which cloud cover estimates (from method 2) can be made as a function of $R$ and true cloud cover. 
cloud covers less than 60 percent, the estimated and true percentages of cloud cover are in close agreement (within 4 percent). To come within 4 percent for cloud covers greater than 70 percent requires an $R$ of about 20. Therefore, for reasonable agreement between the estimated and true cloud-cover percentages for cloud patterns 1 and 2, the second method of cloud cover estimation requires less sensor spatial resolution than does the first method. For $R=1$, within the range of true cloud covers from 30 to 78 percent, the estimated cloud cover was 50 percent, which reflects the dominant role of partially cloud-filled resolution elements.

The nomogram for cloud pattern 3 is shown in Figure 11. This nomogram is nearly identical to the nomogram in Figure 10, except that $R \leqslant 25$ in the cloud cover range from 35 to 78 percent. The estimated cloud covers below $R=0.4$ for 10 and 90 percent true cloud cover are based on extrapolation. The same is true for $R>50$ for most of the nomogram, but the confidence level for this extrapolation is high, since at $R=50$, the estimated percentages of cloud cover are close to the true percentages of cloud cover, especially for true cloud covers less than 40 percent and greater than 60 percent.

These two nomograms can be used in the same manner as the earlier nomogram in Figure 7, associated with the first method of estimation of the percentage of cloud cover. Use of Figures 10 and 11 requires a rough estimate of $R$ as well as a cloud/no-cloud brightness threshold at the low end of the brightness range and a means of ascertaining that a resolution element is completely cloud filled. With this information, an estimated percentage of cloud cover can be corrected to a value that is probably closer to the true percentage of cloud cover. The most important portion of the curves is from $R=1$ to $R=25$. For $R>25$, the estimated percentage of cloud cover is very close to the true percentage of

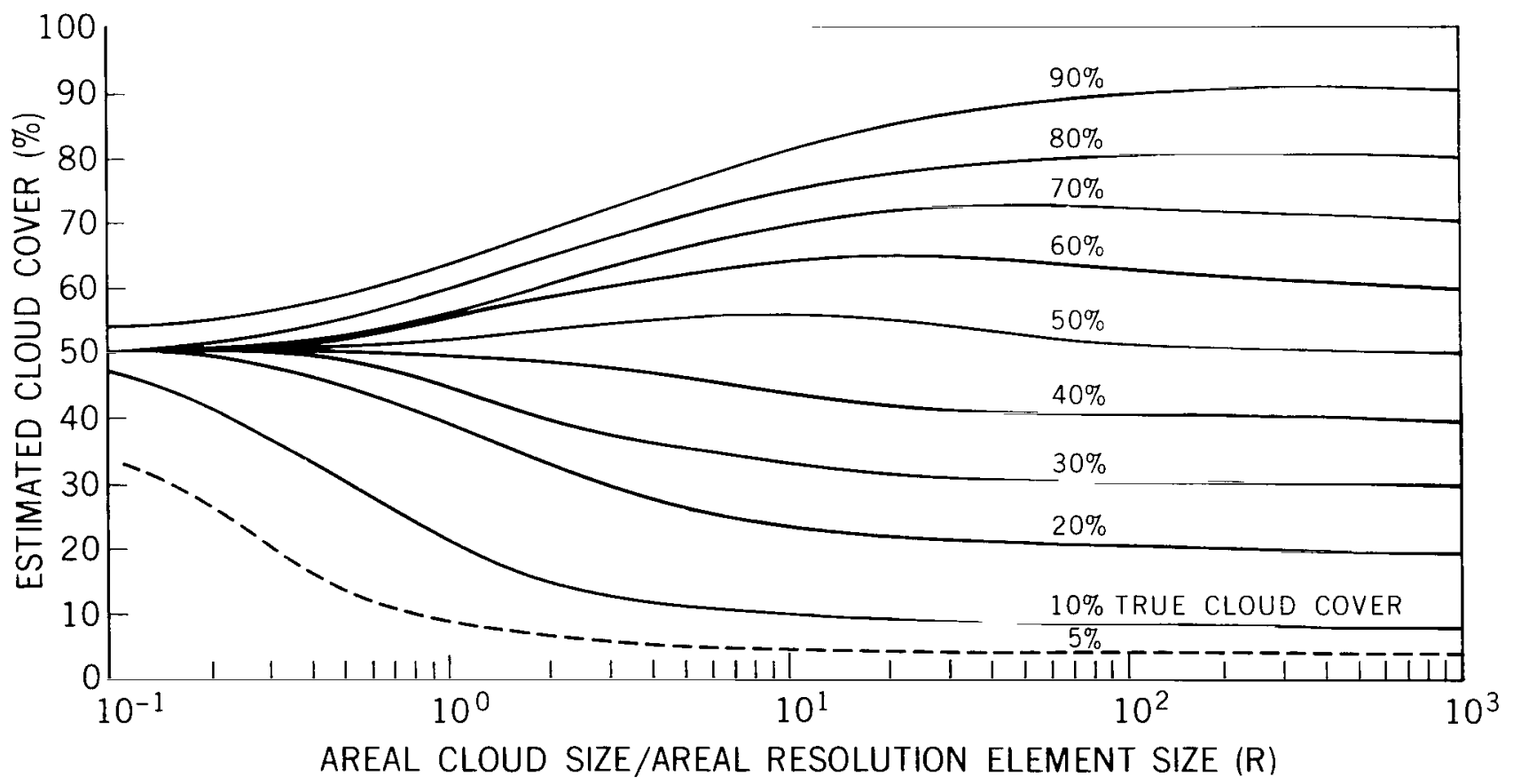

Figure 11-A nomogram generated from simulated cloud pattern 3, from which cloud cover estimates (from method 2) can be made as a function of $R$ and true cloud cover. 
cloud cover. When $R<1$, a small error in the estimated percentages of cloud cover near 50 percent results in a substantial error in the determined true percentage of cloud cover.

\section{Satellite Data Test}

Since all of the cloud patterns considered so far were simulated, the evaluation of a real cloud pattern was thought to be desirable. This real data sample needed the following characteristics: (1) High spatial resolution, so that each cloud element would be readily discernible and, therefore, the measured percentage of cloud cover would be very close to the true percentage of cloud cover, and (2) high altitude sensor location such that an extensive cloud pattern could be viewed almost vertically with only small perspective differences within the area. The photography from the flight of Apollo 6 satisfied these requirements, since the spatial resolution was, conservatively, about $100 \mathrm{~m}$ and the local zenith angle was within $21^{\circ}$ at the edge of each picture. Frame 968, taken over Africa, was selected for analysis. Two overlapping cloud fields were extracted from this picture, and the cloud pattern from one of these is shown in Figure 12. The predominant cloud type in both real cloud fields was cumulus, with a few cirrus streaks present. As can be seen in Figure 12, the general nature of the real cloud pattern was similar to simulated cloud pattern 3 . Since the picture was taken in the early afternoon (13:58 solar time) over a tropical area, convective processes may account for the cumuliform clouds observed. Lake Volta is present in the clear corner of Figure 12. This lake region was devoid

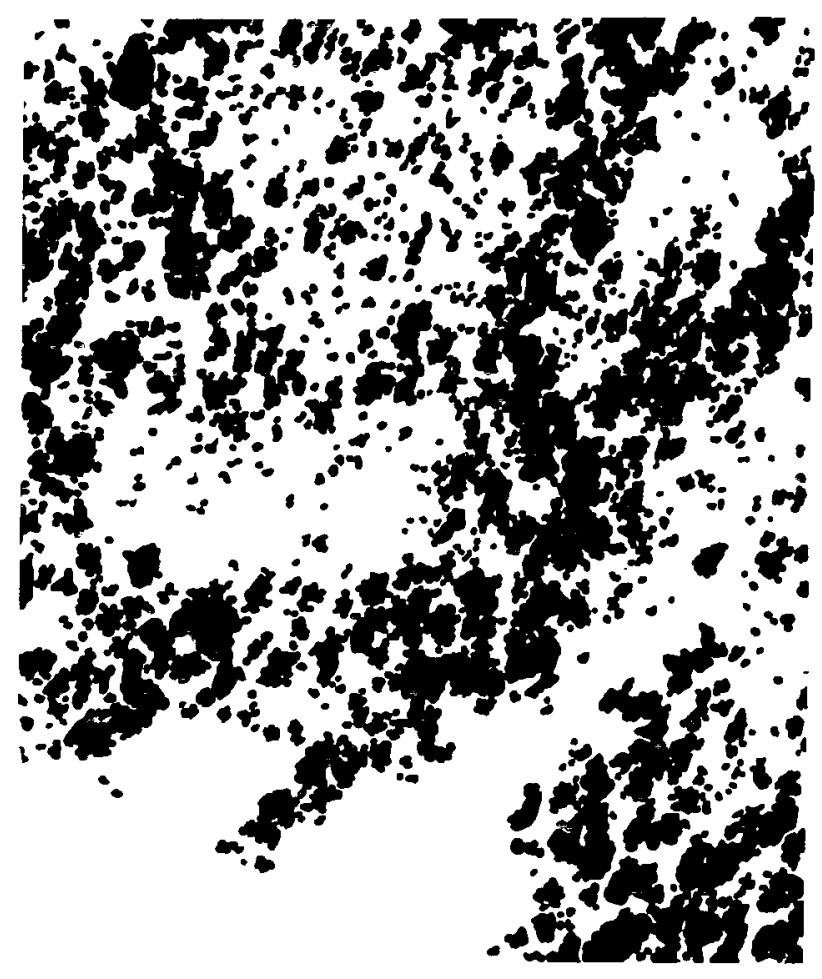

Figure 12-Cloud field (dark areas are clouds) extracted from a portion of a photograph (Frame 968) taken on the Apollo 6 mission, April 4, 1968. of cumulus activity due to insufficient heating of the water necessary to produce convective instability. Cumulus clouds appeared throughout the cloud pattern not shown in Figure 12, as the camera was aligned such that a portion of Lake Volta was not included. The area covered by both Apollo cloud fields is approximately $7000 \mathrm{~km}^{2}$.

Cloud-cover percentages were determined from these two real cloud fields by the same methods that were employed from the three simulated cloud patterns. The most important range of $R$ was covered (from about 0.15 to 20 ). Table 2 lists the $R$ values and the cloud-cover percentages computed by each method for the two Apollo 6 cloud fields. The $R$ values for each Apollo 6 cloud field are about the same as would be expected for cloud fields that largely overlap. The true cloud cover for the cloud field containing Lake Volta was 37.2 percent, whereas the true cloud cover for the other cloud field was 40.8 percent. Table 2 indicates that the estimates of method 2 converged quickly toward the true 
Table 2-Values of $R$ and the percentages of cloud cover for the two Apollo 6 cloud fields.

\begin{tabular}{|c|c|c|c|c|c|c|c|}
\hline \\
\hline \multicolumn{8}{|c|}{ Cloud field 1 (including Lake Volta) } \\
\hline & \multicolumn{7}{|c|}{ Resolution element grid } \\
\hline & $10 \times 15$ & $20 \times 30$ & $40 \times 60$ & $60 \times 90$ & $80 \times 120$ & $100 \times 150$ & $120 \times 180$ \\
\hline$R$ & 0.11 & 0.45 & 1.81 & 4.05 & 7.19 & 11.22 & 16.23 \\
\hline Cloud cover (method 1) (\%) & 93.3 & 87.8 & 78.7 & 71.1 & 62.9 & 60.2 & 57.3 \\
\hline Cloud cover (method 2) (\%) & 46.7 & 43.9 & 40.6 & 38.9 & 37.6 & 37.5 & 37.7 \\
\hline \multicolumn{8}{|c|}{ Cloud field 2 (excluding Lake Volta) } \\
\hline & \multicolumn{7}{|c|}{ Resolution element grid } \\
\hline & $10 \times 15$ & $20 \times 30$ & $40 \times 60$ & $60 \times 90$ & $80 \times 120$ & $100 \times 150$ & $120 \times 180$ \\
\hline$R$ & 0.12 & 0.48 & 1.94 & 4.37 & 7.75 & 12.10 & 17.50 \\
\hline Cloud cover (method 1) (\%) & 100.0 & 96.3 & 83.0 & 75.5 & 69.9 & 65.2 & 61.2 \\
\hline Cloud cover (method 2) (\%) & 50.0 & 48.2 & 43.8 & 42.8 & 42.6 & 42.4 & 41.1 \\
\hline
\end{tabular}

percentage of cloud cover for both cloud fields. As might be expected, the cloud-cover percentage error for method 1 was still substantial for $R$ values of approximately 15 . These results were generally consistent with those found with the simulated cloud patterns. To further explore the compatibility of the real and simulated cloud pattern results, two curves were prepared that compare the Apollo 6 estimated cloud-cover percentages with the cloud cover percentages determined from the simulated cloud patterns for both methods. Figures 13 and 14 depict the cloud-cover percentages estimated by method 1 as a function of $R$ for Apollo 6 cloud fields 1 and 2, respectively. Also indicated in Figures 13 and 14 are the appropriate curves for the true cloud cover as a function of $R$, as determined from the simulated cloud patterns (from Figure 7). Comparison of the two curves in Figure 13 reveals that the agreement improved with increasing $R$ such that when $R=10$ there was less than a factor of 2 difference in $R$ for the same estimated cloud-cover percentage. For a given $R$, the differences in estimated cloud cover varied between 4 and 14 percent; the greatest difference was associated with the smaller $R$ values. A logical explanation for the differences between the two curves in Figure 13 is the presence of the large clear area over Lake Volta and other large nearly clear areas almost in the center and in the upper left hand corner of the cloud field (Figure 12). No large clear areas appeared in the simulated cloud patterns, except for the small percentages of cloud cover. As a result, the large clear areas were correctly identified as clear areas even with the large resolution elements in the Apollo 6 cloud field shown in Figure 12. Therefore, the estimation difference between the real and simulated cloud patterns was from about 10 to 14 percent for small values of $R$.

The results of the comparison between the real and simulated data in Figure 14 are generally the same as those for Figure 13. Due to the absence of the large clear area over the lake, the estimated cloud-cover percentage is higher for small $R$ values in Figure 14 than in Figure 13. However, the other 


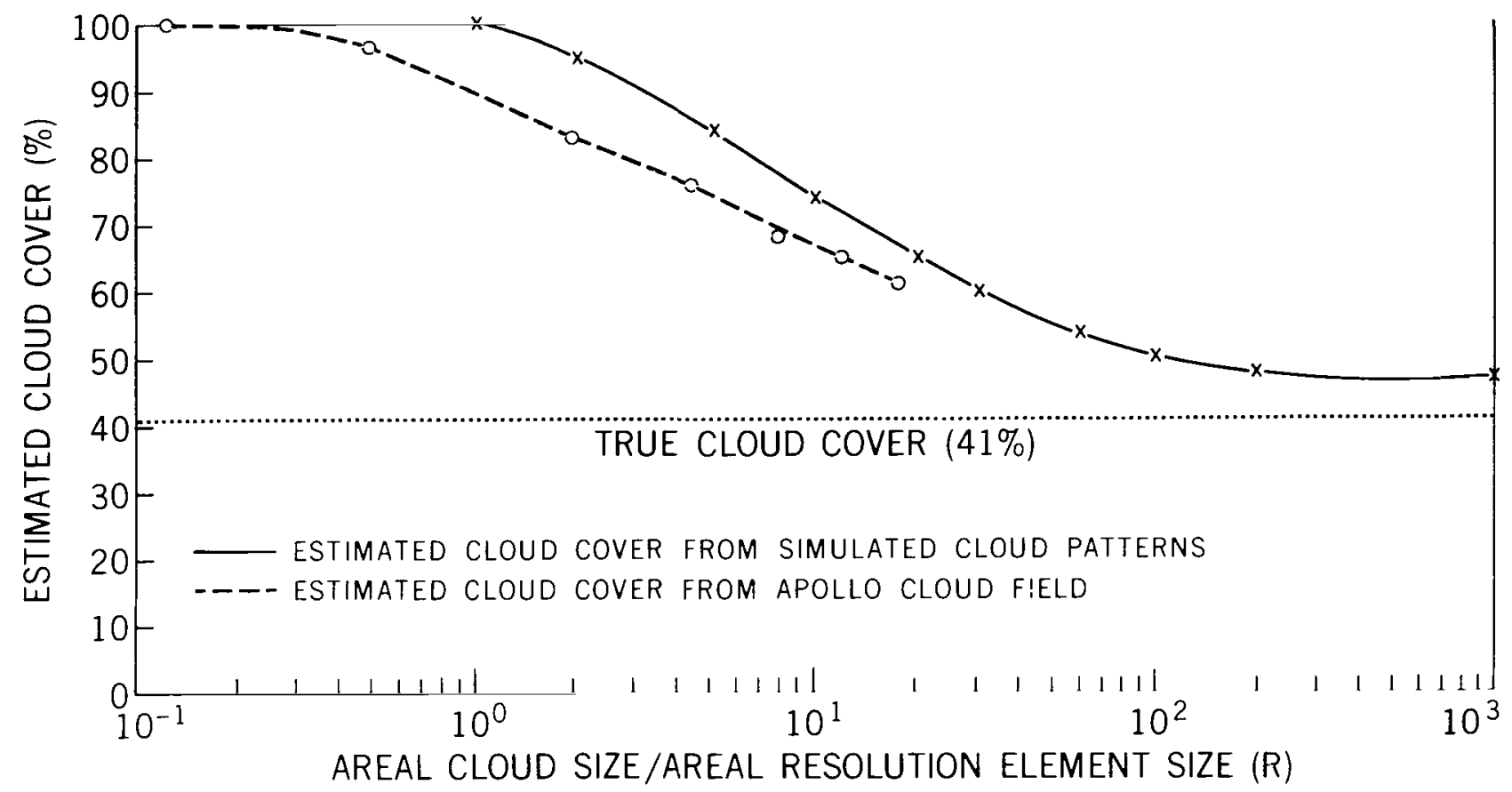

Figure 13-Comparison of method 1 estimated cloud cover (from Figure 7) with the cloud cover determined from Apollo cloud field 1 as a function of $R$.

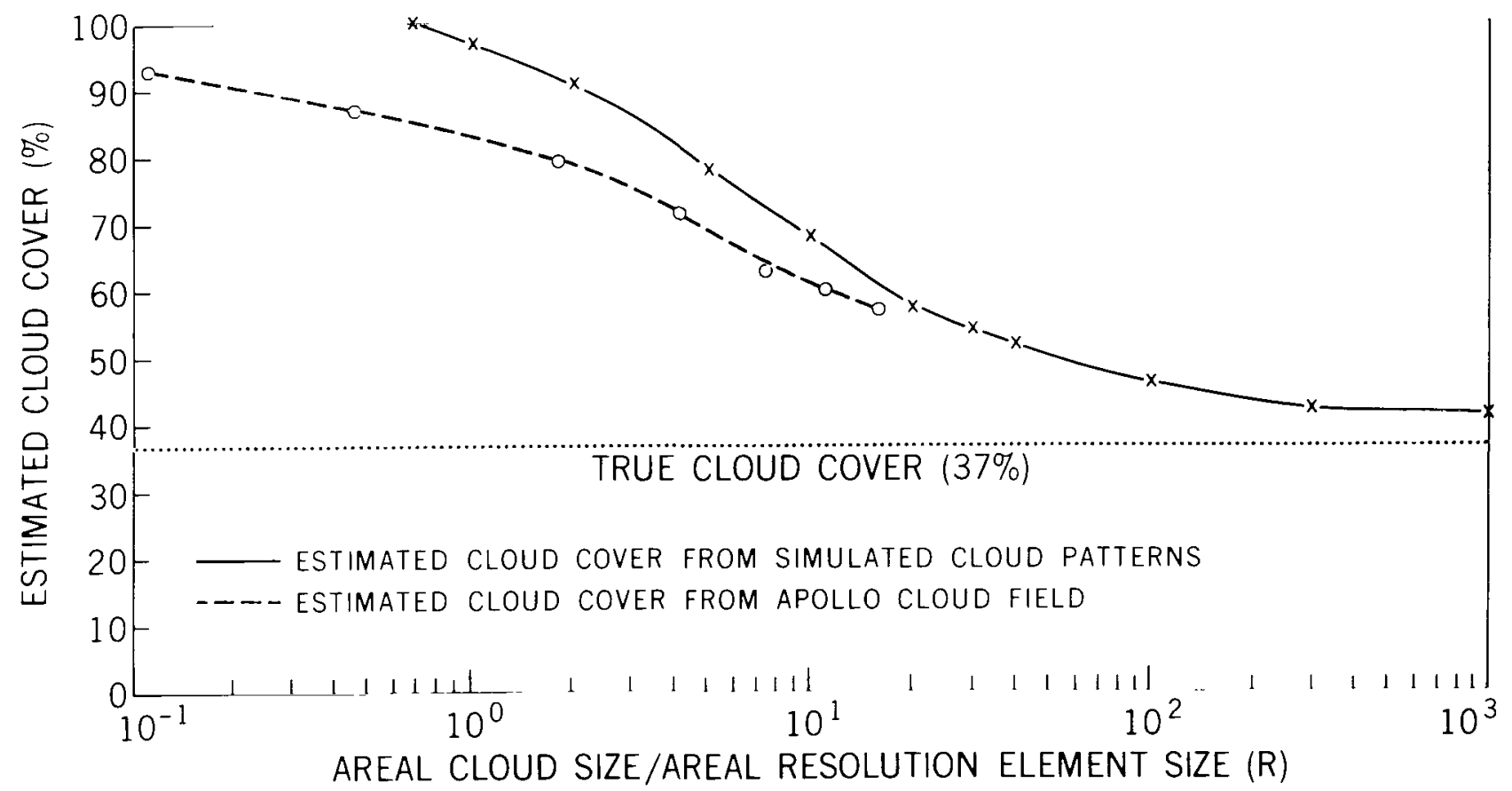

Figure 14-Comparison of method 1 estimated cloud cover (from Figure 7) with the cloud cover determined from Apollo cloud field 2 as a function of $R$. 
large, relatively clear areas that appeared in Figure 12 were also present in Apollo 6 cloud field 2 and contributed in a similar manner to the cloud cover estimates, which were smaller for a given $R$ than the estimates from the simulated cloud patterns. From a practical viewpoint, it is unnecessary to apply cloud-cover determination methods to clear areas that are considerably larger than a resolution element. Thus, at least some of the discrepancies noticed between the real and simulated estimated cloud-cover percentage curves would probably be eliminated if these large clear regions were isolated from the rest of the data. Also, the differences between the simulated and real cloud pattern curves shown in Figures 13 and 14 are probably within the accuracy of estimations of $R$ made from a real cloud pattern without having independent detailed information on cloud size.

The cloud-cover percentages determined by method 2 from the two Apollo 6 cloud fields were compared with the comparable data from the simulated cloud patterns (Figures 15 and 16). Figures 15 and 16 are similar in content to Figures 13 and 14, where, in this case, the Apollo 6 method 2 cloud-cover percentages over a range of $R$ are compared with the method 2 cloud-cover percentage curves developed for simulated cloud pattern 3. The cloud-cover percentages estimated from the two real cloud fields begin to approach the true cloud-cover percentage at smaller values of $R$ than do those

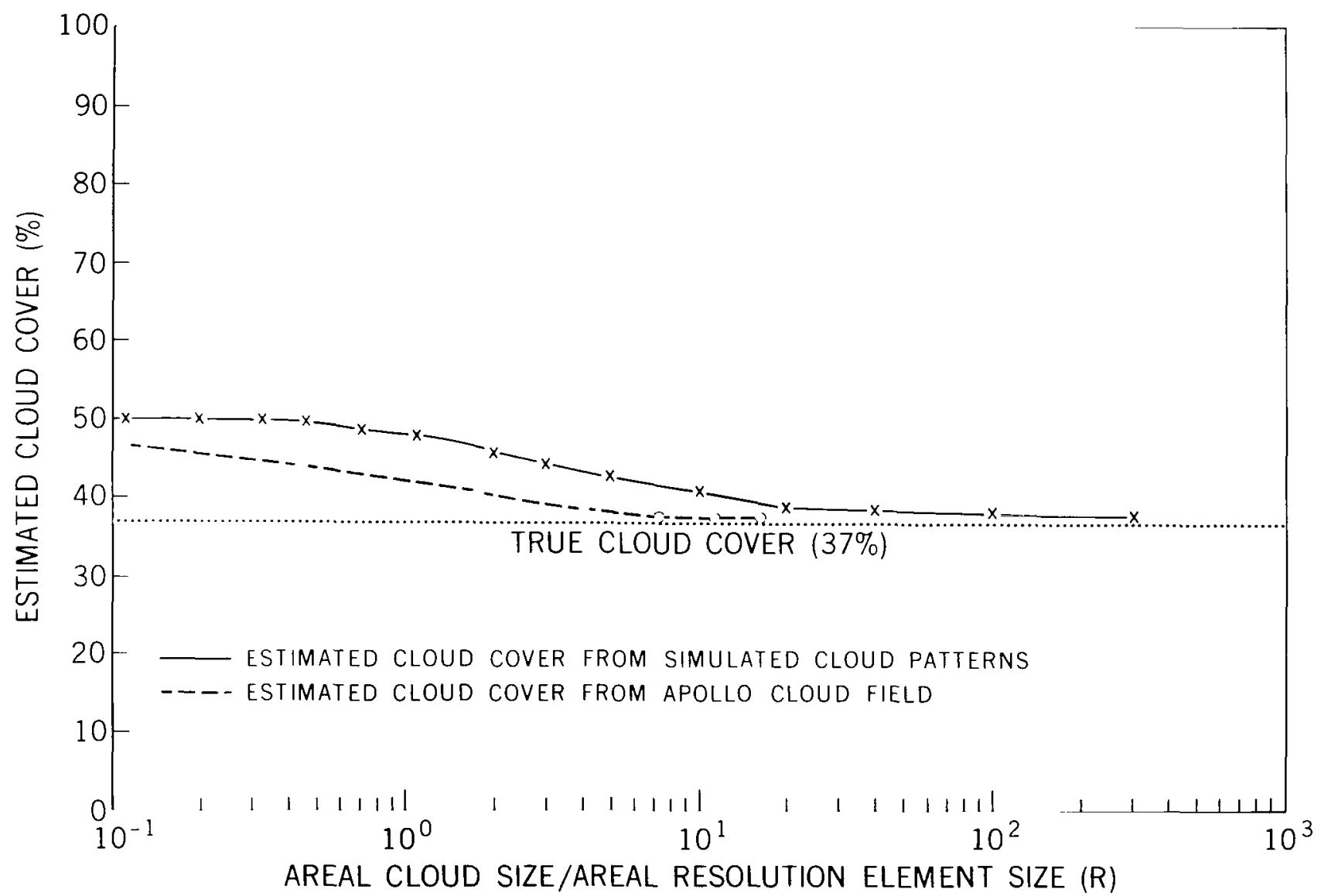

Figure 15-Comparison of method 2 estimated cloud cover (from Figure 11) with the cloud cover determined from Apollo cloud field $l$ as a function of $R$. 


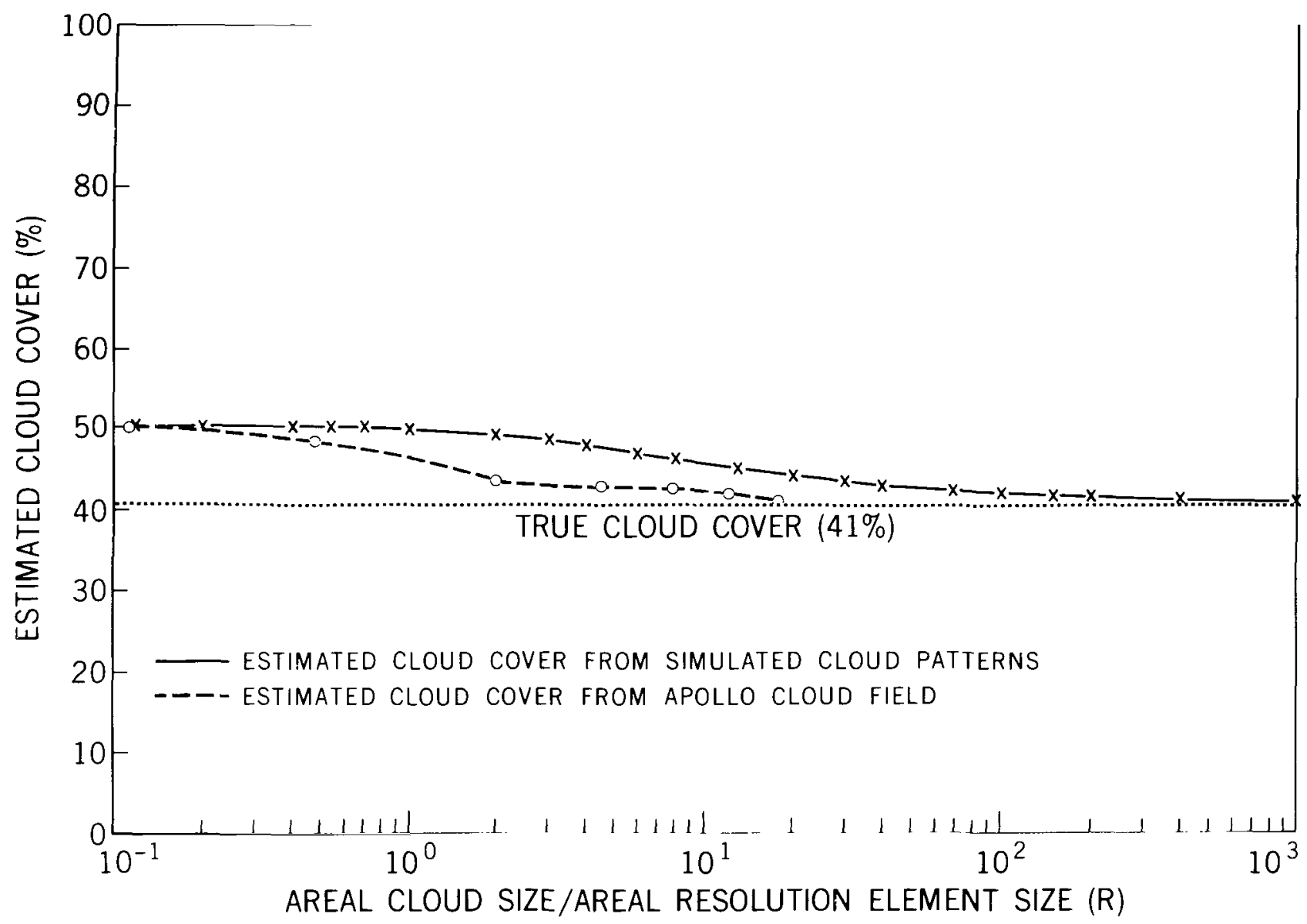

Figure 16-Comparison of method 2 estimated cloud cover (from Figure 11) with the cloud cover determined from Apollo cloud field 2 as a function of $R$.

from the simulated cloud patterns. Again it is hypothesized that the relatively large clear areas permit better estimates of the cloud-cover percentage from the Apollo 6 cloud fields for a given $R$ due to the correct identification of the clear areas with large resolution elements. In Figures 15 and 16, for $R>10$, the real and simulated cloud-cover estimates are within 5 percent of the true cloud covers.

The average cloud size of one of the Apollo 6 cloud fields was computed and the cloud-cover percentage was estimated, based on an $R$ value determined by a resolution element size that is currently available on operational meteorological satellites. The average cloud size of Apollo 6 cloud field 1 (Lake Volta) was $5.25 \mathrm{~km}^{2}$, and the appropriate resolution element size used was $13.69 \mathrm{~km}^{2}(3.7 \mathrm{~km}$ square resolution element). Therefore, the calculated $R$ value is 0.38 . From Figure 13 , the cloud covers estimated by method 1 were 89 and 100 percent, respectively, from the real and simulated data curves; the cloud covers determined by method 2 (Figure 15) were 44 and 50 percent for the real and simulated cloud patterns, respectively. Thus, the cloud-cover percentages calculated by method 2 were the most accurate. If the spatial resolution of the satellite sensor were improved to $0.9 \mathrm{~km}$ (the highest visible resolution currently planned for flight on a meteorological spacecraft) the value of $R$ would increase to 6.5 . Then the cloud covers estimated by method 1 would be 65 and 74 percent for 
the real cloud field and the simulated cloud patterns, respectively, and in the same order the method 2 estimated cloud covers would be 42 and 38 percent. These results are improvements over the $3.7-\mathrm{km}$ resolution, but, in the case of method 1 , they are still far from the true cloud-cover percentages. It is apparent from this analysis that the nomograms in Figures 7, 10, and 11 can improve cloud-cover determinations if $R$ is known with fair precision. The selected Apollo 6 cloud fields were considered to be reasonably representative of cumuliform cloud fields that are very prevalent in the tropics and the subtropics.

\section{CONCLUSIONS}

Sensor spatial resolution has a strong effect on estimates of the percentage of cloud cover. It is concluded that $R$ values of about 100 or greater are required with a one cloud/no-cloud brightness threshold technique (method 1) to measure directly the cloud cover to within approximately 10 percent, the accuracy decreasing with increasing cloud-cover percentage. However, with some knowledge of $R$ and an estimated cloud-cover percentage, it is possible to improve substantially the cloud-cover estimates with the nomogram in Figure 7. A test of this nomogram with real cloud data verified the usefulness of the nomogram, especially if large clear areas were excluded. An analysis of the real data with a sensor spatial resolution in common use on meteorological satellites revealed that $R$ is probably less than 1 over substantial regions of the tropics and subtropics. There, the percentages of cloud cover would be considerably overestimated.

The cloud-cover estimates improved for a given $R$ when method 2 was applied. This two threshold method requires $R \geqslant 10$ for good cloud-cover estimate results. Unlike method 1 , method 2 needs $R \geqslant 1$ before the nomograms can be effectively used to improve cloud-cover estimates due to the convergence of the true percentage of cloud-cover curves to near 50 percent for $R \leqslant 1$.

Future improvements in cloud-cover percentage estimation can be made by the determination of $R$ for various cloud regimes, better sensor spatial resolutions (resulting in larger $R$ values), and development of improved cloud/no-cloud thresholds with instruments of sufficient sensitivity. Also, quantitative assessments of the viewing perspective effect are needed with improved models and with real cloud data.

\section{ACKNOWLEDGMENT}

The authors applaud the tireless effort of Mr. Mark Smith, who assessed the state of cloudiness in over two million resolution elements.

\footnotetext{
Goddard Space Flight Center

National Aeronautics and Space Administration

Greenbelt, Maryland, October 14, 1970

160-44-51-01-51
} 


\section{REFERENCES}

Brown, S. C., "Simulating the Consequence of Cloud Cover on Earth-Viewing Space Missions," Bull. Amer. Meteorol. Soc. 51:126-131, 1970.

Conover, J. H., "Cloud Interpretation from Satellite Attitudes," Air Force Cambridge Research Laboratories Report AFCRL-62-680, Cambridge Research Laboratories, Bedford, Mass., 77 pp., 1962.

Conover, J. H., "Cloud Interpretation from Satellite Attitudes," Air Force Cambridge Research Laboratories Report AFCRL-62-680 Suppl. 1, Cambridge Research Laboratories, Bedford, Mass., 19 pp., 1963.

Hanel, R., and Conrath, B., "Interferometer Experiment on Nimbus 3: Preliminary Results," Science 165:1258-1260, 1969.

Sherr, P. E., Glaser, A. H., Barnes, J. C., and Willand, J. H., "World-Wide Cloud Cover Distributions for Use in Computer Simulations," NASA Contractor Report 61226, National Aeronautics and Space Administration, Washington, D. C., 140 pp., 1968.

Stamm, A. J., and Von der Haar, T. H., "A Study of Cloud Distribution Using Reflected Radiance Measurements from ATS Satellites," J. Appl. Meteorol. 9:498-507, 1970.

Wark, D. Q., and Hilleary, D. T., "Atmospheric Temperature: Successful Test of Remote Probing," Science 165:1256-1258, 1969.

Young, M. J., "Variability in Estimating Total Cloud Cover From Satellite Pictures," J. Appl. Meteorol. 6(3):573-579, 1967. 


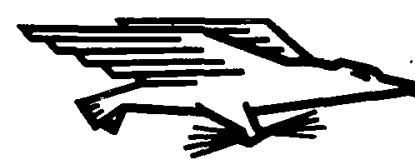

POSTAGE AND FEES PAID NATIONAL AERONAUTICS AND SPACE ADMINISTRATION

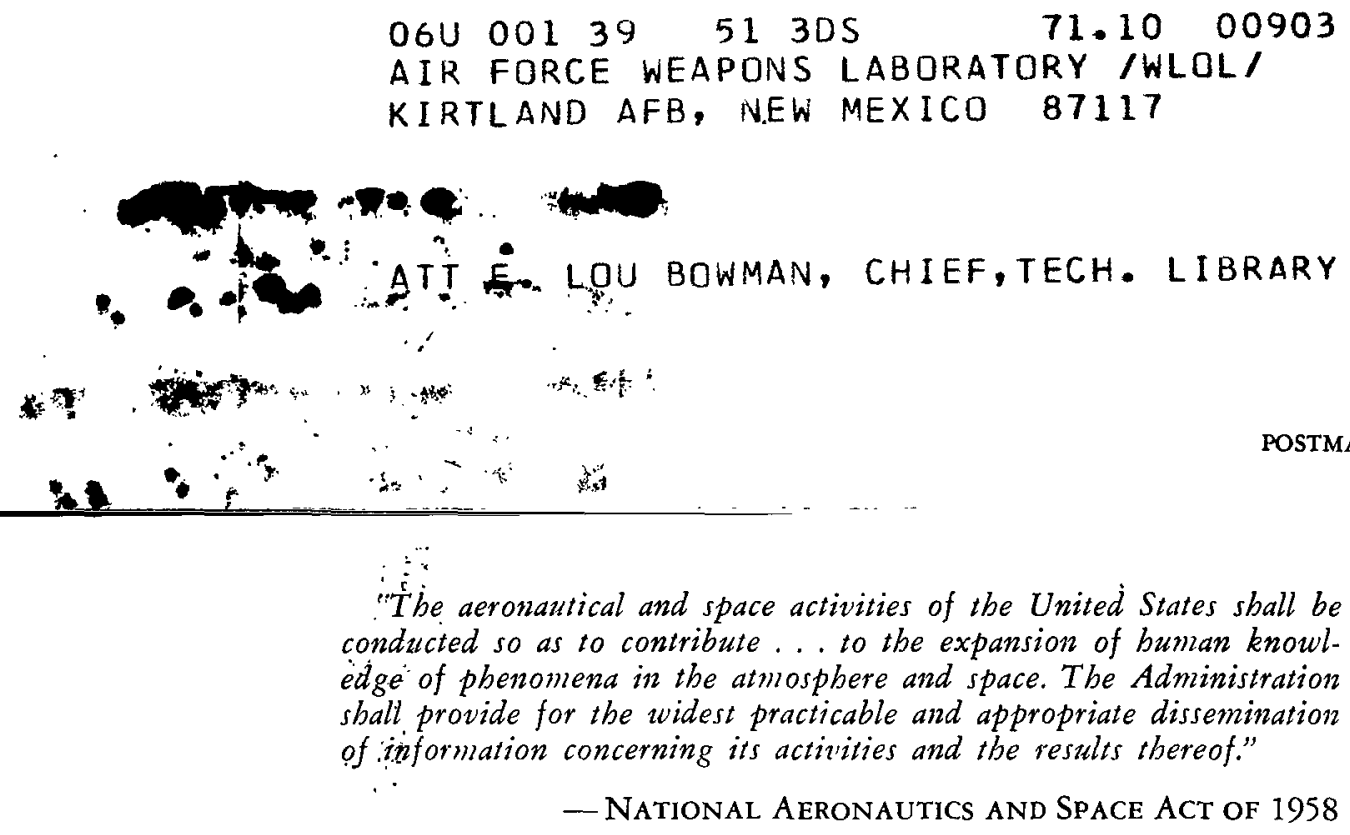

\section{NASA SCIENTIFIC AND TECHNICAL PUBLICATIONS}

TECHNICAL REPORTS: Scientific and technical information considered important, complete; and a lasting contribution to existing knowledge. ' .

TECHNICAL NOTES: Information less broad in scope but nevertheless of importance as a contribution to existing knowledge.

TECHNICAL MEMORANDUMS:

Information receiving limited distribution because of preliminary data, security classification, or other reasons.

CONTRACTOR REPORTS: Scientific and technical information generated under a NASA contract or grant and considered an important contribution to existing knowledge.
TECHNICAL TRANSLATIONS: Information published in a foreign language considered to merit NASA distribution in English.

SPECIAL PUBLICATIONS: Information derived from or of value to NASA activities. Publications include conference proceedings, monographs, data compilations, handbooks, sourcebooks, and special bibliographies.

TECHNOLOGY UTILIZATION PUBLICATIONS: Information on technology used by NASA that may be of particular interest in commercial and other non-aerospace applications. Publications include Tech Briefs, Technology Utilization Reports and Technology Surveys.

Details on the availability of these publications may be obtained from:

SCIENTIFIC AND TECHNICAL INFORMATION OFFICE NATIONAL AERONAUTICS AND SPACE ADMINISTRATION Washington, D.C. 20546 